\title{
Three-cocycles, Nonassociative Gauge Transformations and Dirac's Monopole
}

\author{
Alexander I. Nesterov* \\ Departamento de Física, CUCEI, Universidad de Guadalajara, \\ Av. Revolución 1500, Guadalajara, CP 44420, Jalisco, México
}

(Dated:)

\begin{abstract}
The relation between 3-cocycles arising in the Dirac monopole problem and nonassociative gauge transformations is studied. It is shown that nonassociative extension of the group $\mathrm{U}(1)$ allows to obtain a consistent theory of pointlike magnetic monopole with an arbitrary magnetic charge.

PACS numbers: 14.80.Hv, 03.65.-w, 03.50.De,05.30.Pr, 11.15.-q,

Keywords: cocycle, monopole, nonassociativity, gauge loop, quasigroup
\end{abstract}

\section{INTRODUCTION}

In 1931 Dirac [1] introduced a magnetic monopole into the quantum mechanics and found a quantization relation between an electric charge $e$ and magnetic charge $q$, $2 \mu=n, n \in \mathbb{Z}$, where $\mu=e q$, and $\hbar=c=1$. One of the widely accepted proofs of the Dirac selection rule is based on group representation theory (see, for example, 2, 3, 4, 5, 6]).

In the presence of the magnetic monopole the operator of the total angular momentum J, which uncludes contribution of the electromagnetic field, obeys the standard commutation relations of the Lie algebra of the rotation group

$$
\left[J_{i}, J_{j}\right]=i \epsilon_{i j k} J_{k}
$$

and this is true for any value of $\mu$. Notice that these commutation relations fail on the Dirac string, restricting the domain of definition of the operator $\mathbf{J}$ and limiting it to the functions that vanish sufficiently rapidly on the string 4]. Here $\mathbf{J}$ may be extended to self-adjoint operator satisfying the commutation relations of the rotation group for an arbitrary $\mu$ [5].

The requirement that $J_{i}$ generate a finite-dimensional representation of the rotation group yields $2 \mu$ being integer and only values $2 \mu=0, \pm 1, \pm 2, \ldots$ are allowed. However, employing infinite-dimensional representations of the rotation group one can relax Dirac's condition and obtain the consistent monopole theory with an arbitrary magnetic charge $7,8,9]$.

The situation with the translations in the background of the monopole is completelely different from the rotations. Since the Jacobi identity fails for the gauge invariant algebra of translations they do not form the Lie algebra, and for the finite translations one has 10, 11, 12, 13.

$$
\left(U_{\mathbf{a}} U_{\mathbf{b}}\right) U_{\mathbf{c}} \Psi(\mathbf{r})=\mathrm{e}^{i \alpha_{3}(\mathbf{r} ; \mathbf{a}, \mathbf{b}, \mathbf{c})} U_{\mathbf{a}}\left(U_{\mathbf{b}} U_{\mathbf{c}}\right) \Psi(\mathbf{r})
$$

where $\alpha_{3}$ is the so-called 3-cocycle. For the Dirac quantization condition being satisfied, one has $\alpha_{3}=0$

*Electronic address: nesterov@cencar.udg.mx $\bmod (2 \pi n)$; and Eq.(1) provides an associative representation of the translations, in spite of the fact that the Jacobi identity for the infinitesimal generators continues to fail. The 3-cocycles, besides being appeared in the Dirac monopole background, lead also to a nonassociative algebra of defects in quantum-mechanical systems and are related to axions in string theory [14, 15]. The low-dimensional cocycles are known also in modern physics in relation with the non-Abelian anomalies and Wess-Zumino action. For instance, the Wess-Zumino functional is identified as 1-cocycle and the anomalous Schwinger term as a 2-cocycle 16, 17, 18.

In this letter we introduce nonassociative gauge transformations and give a new interpretation of 3-cocycle linking it with the associator of the gauge loop. We argue that a nonassociative extension of the conventional group U(1) allows an arbitrary magnetic charge.

\section{COCYCLES AND MAGNETIC MONOPOLE}

Following [17] consider a group $G$ acting on a manifold $\mathfrak{M}$ as a group of transformations: $x \rightarrow x g, x \in \mathfrak{M}, g \in$ $G$. For an array function $\alpha_{n}\left(x ; g_{1}, \ldots, g_{n}\right)$, called the $n$-cochain, the coboundary operator $\delta$ is defined as

$$
\begin{aligned}
& \left(\delta \alpha_{n}\right)\left(x ; g_{1}, g_{2}, \ldots, g_{n+1}\right)=\alpha_{n}\left(x g_{1} ; g_{2}, \ldots, g_{n+1}\right) \\
& -\alpha_{n}\left(x ; g_{1} g_{2}, \ldots, g_{n+1}\right)+\ldots \\
& +(-1)^{i} \alpha_{n}\left(x ; g_{1}, \ldots, g_{i} g_{i+1}, \ldots, g_{n+1}\right) \\
& +(-1)^{n+1} \alpha_{n}\left(x ; g_{1}, \ldots, g_{n}\right)
\end{aligned}
$$

and it is easy to check that $\delta^{2}=0$. A cochain $\alpha_{n}=\delta \alpha_{n}$ is called a coboundary, and if $\alpha_{n}$ satisfies $\delta \alpha_{n}=0$, it is called a cocycle. A cocycle is nontrivial if it is not a coboundary, i.e. $\delta \alpha=0$, but $\alpha \neq \delta \beta$. Notice, that the $n$-cochain can be associated with a differential $n$-form $\boldsymbol{\omega}_{n}$ as

$$
\alpha_{n}\left(x ; g_{1}, g_{2}, \ldots, g_{n}\right)=\int_{\Sigma_{n}} \omega_{n},
$$

where $\Sigma_{n}$ is a $n$-simplex with vertices $\left(x, x g_{1}, \ldots, x g_{1} \ldots g_{n}\right)$.

The 1-cochain $\alpha_{1}(x ; g)$ appears in the representation $U_{g}$ of the group $G$ in the space of the functions $\Psi(x)$ on 
$\mathfrak{M}:$

$$
U_{g} \Psi(x)=\mathrm{e}^{i \alpha_{1}(x ; g)} \Psi(x g) .
$$

If $\alpha_{1}(x ; g)$ is a trivial cocycle,

$$
\alpha_{1}(x ; g)=\delta \alpha_{0}(x ; g)=\alpha_{0}(x g)-\alpha_{0}(x),
$$

then the representation reduces to the usual one

$$
U_{g_{1}} U_{g_{2}} \Psi(x)=U_{g_{1} g_{2}} \Psi(x),
$$

by the unitary transformation $\Psi(x) \mapsto \exp \left(i \alpha_{0}(x)\right) \Psi(x)$. by

A 2-cocycle $\alpha_{2}=\delta \alpha_{1}$ defined for a given 1-cochain $\alpha_{1}$

$$
\alpha_{2}\left(x ; g_{1}, g_{2}\right)=\alpha_{1}\left(x g_{1} ; g_{2}\right)-\alpha_{1}\left(x ; g_{1} g_{2}\right)+\alpha_{1}\left(x ; g_{1}\right)
$$

appears in the product of two operators:

$$
U_{g_{1}} U_{g_{2}} \Psi(x)=\mathrm{e}^{i \alpha_{2}\left(x ; g_{1}, g_{2}\right)} U_{g_{1} g_{2}} \Psi(x) .
$$

When the 2-cocycle does not depend on $x$, Eq. (8) provides a projective representation of the group $G$, i.e.

$$
U_{g_{1}} U_{g_{2}}=\mathrm{e}^{i \alpha_{2}\left(g_{1}, g_{2}\right)} U_{g_{1} g_{2}} .
$$

The condition of associativity for (9) yields $\alpha_{3}=\delta \alpha_{2}=0$, and for $\alpha_{2}$ being a trivial 2-cocycle the representation (9) reduces to an ordinary one.

Let $\alpha_{2}\left(x ; g_{1}, g_{2}\right)$ be a 2 -cochain, then the 3 -cocycle $\alpha_{3}=\delta \alpha_{2}$, is given by

$$
\begin{aligned}
\alpha_{3}\left(x ; g_{1}, g_{2}, g_{3}\right)= & \alpha_{2}\left(x g_{1} ; g_{2}, g_{3}\right)-\alpha_{2}\left(x ; g_{1} g_{2}, g_{3}\right)+ \\
& +\alpha_{2}\left(x ; g_{1}, g_{2} g_{3}\right)-\alpha_{2}\left(x ; g_{1}, g_{2}\right) .
\end{aligned}
$$

The 3-cocycle enters in the triple-product in the similar way as $\alpha_{2}$ appears in the product of two operators:

$$
\left(U_{g_{1}} U_{g_{2}}\right) U_{g_{3}}=\mathrm{e}^{i \alpha_{3}\left(x ; g_{1}, g_{2}, g_{3}\right)} U_{g_{1}}\left(U_{g_{2}} U_{g_{3}}\right) .
$$

Associativity is fulfilled if $\alpha_{3}=0 \bmod (2 \pi n), n \in \mathbb{Z}$, and we see that the 3-cocycle measures the lack of associativity.

In what follows we consider a generalization of the translation group related with the cocycles and string group [19]. The string group, denoted as String $\mathfrak{M}$, is the group of all paths $\gamma:[0,1] \mapsto$ Diff $\mathfrak{M}$, where Diff $\mathfrak{M}$ denotes the diffeomorphism group on $\mathfrak{M}=R^{3} \backslash\{0\}$ such that $\mathbf{r} \mapsto \mathbf{r}(t)=\mathbf{r} \gamma(t), t \in[0,1]$ and $\gamma(0)=$ identity; the group composition is defined as $\gamma_{12}(t)=\gamma_{1}(t) \gamma_{2}(t)$.

In the presence of magnetic monopole we define the 1-cochain $\alpha_{1}$ as

$$
\alpha_{1}(\mathbf{r} ; \gamma)=e \int_{\mathbf{r}}^{\mathbf{r}^{\prime}} \mathbf{A}(\boldsymbol{\xi}) \cdot d \boldsymbol{\xi}
$$

where the integration is performed along a path $\gamma$ connecting a point $\mathbf{r}$ with a point $\mathbf{r}^{\prime}=\mathbf{r} \gamma(1)$, and $\mathbf{A}(\mathbf{r})$ is the vector potential. If the integral does not depend on the path, one has

$$
\alpha_{1}=\delta \alpha_{0}=\alpha_{0}(\mathbf{r})-\alpha_{0}\left(\mathbf{r}^{\prime}\right),
$$

and $\alpha_{1}$ is the trivial 1-cocycle.

Following 10, 11, 13, 19] we introduce

$$
\alpha_{2}\left(\mathbf{r} ; \gamma_{1}, \gamma_{2}\right)=e \int_{\Sigma} \mathbf{B} \cdot d \mathbf{S}=\left.e \Phi\right|_{\Sigma}
$$

where $\left.\Phi\right|_{\Sigma}$ is a magnetic flux through the twodimensional simplex $\Sigma$ defined as follows: The surface is parametrized as $\mathbf{r}(t, s)=\mathbf{r} \gamma_{1}(t) \gamma_{2}(s)$ with $0 \leq s \leq t \leq 1$, and the vertices are $\left(\mathbf{r}, \mathbf{r}_{1}, \mathbf{r}_{2}\right)$, where $\mathbf{r}_{1}=\mathbf{r} \gamma_{1}(1)$ and $\mathbf{r}_{2}=\mathbf{r} \gamma_{2}(1)$.

Notice that any choice of the vector potential $\mathbf{A}$ being compatible with a magnetic field $\mathbf{B}=q \mathbf{r} / r^{3}$ of Dirac monopole must have singularity (the so-called Dirac string). This implies $\mathbf{B}=\nabla \times \mathbf{A}$ locally, but not globally, so that $\alpha_{2}$ is a 2 -cochain and not a 2 -cocycle. Thus, one can write

$$
\mathbf{B}=\operatorname{rot} \mathbf{A}+\mathbf{h}
$$

where $\mathbf{h}$ is the magnetic field of the string $\mathcal{C}$. Substituting (14) into (13) and applying Stokes' theorem we get

$$
\alpha_{2}\left(\mathbf{r} ; \gamma_{1}, \gamma_{2}\right)=\delta \alpha_{1}\left(\mathbf{r} ; \gamma_{1}, \gamma_{2}\right)+\sigma(\mathcal{C}, \Sigma)
$$

where

$$
\delta \alpha_{1}=\alpha_{1}\left(\mathbf{r}_{1} ; \gamma_{2}\right)-\alpha_{1}\left(\mathbf{r} ; \gamma_{1} \gamma_{2}\right)+\alpha_{1}\left(\mathbf{r} ; \gamma_{1}\right),
$$

and the contribution $\sigma=e \int_{\Sigma} \mathbf{h} \cdot d \mathbf{S}$ is not zero if and only if the string $\mathcal{C}$ crosses $\Sigma$.

Computation of the 3-cocycle,

$$
\begin{aligned}
& \alpha_{3}\left(\mathbf{r} ; \gamma_{1}, \gamma_{2}, \gamma_{3}\right)=\delta \alpha_{2}=\alpha_{2}\left(\mathbf{r}_{1} ; \gamma_{2}, \gamma_{3}\right)-\alpha_{2}\left(\mathbf{r} ; \gamma_{1} \gamma_{2}, \gamma_{3}\right) \\
& +\alpha_{2}\left(\mathbf{r} ; \gamma_{1}, \gamma_{2} \gamma_{3}\right)-\alpha_{2}\left(\mathbf{r} ; \gamma_{1}, \gamma_{2}\right),
\end{aligned}
$$

yields $\alpha_{3}=4 \pi \mu \bmod (2 \pi n)$ if the monopole is enclosed by the simplex with vertices being $\left(\mathbf{r}, \mathbf{r}_{1}, \mathbf{r}_{2}, \mathbf{r}_{3}\right)$ or zero otherwise.

The cocycles allow to construct the realization of the group String $\mathfrak{M}$ in the space of complex functions on $\mathfrak{M}$ in the following way. Let $\mathcal{G}$ be the gauge group of maps $\lambda_{\gamma}: \mathfrak{M} \mapsto \mathrm{U}(1), \gamma \in$ String $\mathfrak{M}$. Then the extension String ${ }^{g} \mathfrak{M}$ is defined as the product of String $\mathfrak{M} \otimes \mathfrak{M}$ with nonassociative multiplication 19.

$$
\left(\gamma_{1}, \lambda_{\gamma_{1}}\right)\left(\gamma_{2}, \lambda_{\gamma_{2}}\right)=\left(\gamma_{1} \gamma_{2}, \mathcal{A}_{2}\left(\mathbf{r} ; \gamma_{1}, \gamma_{2}\right) \lambda_{\gamma_{12}}\right),
$$

where $\lambda_{\gamma}(\mathbf{r})=\lambda(\mathbf{r} \gamma(1))$, and $\mathcal{A}_{2}$ is a $\mathrm{U}(1)$-valued function on $\mathfrak{M}$ given by $\mathcal{A}_{2}\left(\mathbf{r} ; \gamma_{1}, \gamma_{2}\right)=\exp \left(i \alpha_{2}\left(\mathbf{r} ; \gamma_{1}, \gamma_{2}\right)\right) ; \alpha_{2}$ being the 2-cochain of Eq. (13).

The realization of the group String $\mathfrak{M}$ is defined as follows:

$$
U_{g_{\gamma}} \Psi(\mathbf{r})=\mathcal{A}_{1}(\mathbf{r} ; \gamma) \Psi(\mathbf{r} \gamma), \quad U_{g_{\gamma}} \in \mathrm{U}(1)
$$

where $\mathcal{A}_{1}(\mathbf{r} ; \gamma)=\exp \left(i \alpha_{1}(\mathbf{r} ; \gamma)\right)$, and the composition law for the two operators $\mathcal{A}_{1}$ is taken to be [10, 17]

$$
\mathcal{A}_{1}\left(\mathbf{r} ; \gamma_{1}\right) \mathcal{A}_{1}\left(\mathbf{r} \gamma_{1} ; \gamma_{2}\right)=\mathrm{e}^{i \alpha_{2}\left(\mathbf{r} ; \gamma_{1}, \gamma_{2}\right)} \mathcal{A}_{1}\left(\mathbf{r} ; \gamma_{12}\right) .
$$


The 2-cochain $\alpha_{2}$ enters in the product of the two operators $U_{g_{\gamma}}$ as follows:

$$
U_{g_{\gamma_{1}}} U_{g_{\gamma_{2}}} \Psi(\mathbf{r})=\mathrm{e}^{i \alpha_{2}\left(\mathbf{r} ; \gamma_{1}, \gamma_{2}\right)} U_{g_{\gamma_{12}}} \Psi(\mathbf{r})
$$

The nonassociativity of multiplication is described by the 3-cocycle $\alpha_{3}$ which appears in the triple-product:

$U_{g_{\gamma_{1}}}\left(U_{g_{\gamma_{2}}} U_{g_{\gamma_{3}}}\right) \Psi(\mathbf{r})=\mathrm{e}^{i \alpha_{3}\left(\mathbf{r}, \gamma_{1}, \gamma_{2}, \gamma_{3}\right)}\left(U_{g_{\gamma_{1}}} U_{g_{\gamma_{2}}}\right) U_{g_{\gamma_{3}}} \Psi(\mathbf{r})$.

Just as in Eq. (17) we find that $\alpha_{3}=4 \pi \mu$ if the monopole is enclosed by the simplex with vertices be$\operatorname{ing}\left(\mathbf{r}, \mathbf{r}_{1}, \mathbf{r}_{2}, \mathbf{r}_{3}\right)$ and zero otherwise. The obtained realization is called a nonassociative representation of the group [10]. Associativivty is fulfilled if $\alpha_{3}=\delta \alpha_{2}=0$ $\bmod (2 \pi n)$ and leads to the Dirac's quantization condition, $2 \mu \in \mathbb{Z}$.

\section{NONASSOCIATIVE GAUGE TRANSFORMATIONS}

We start with some elementary facts from the theory of quasigroups and loops (see, e.g. 20, 21, 22] ).

A set $\langle Q, \cdot\rangle$ with a binary operation $(a, b) \mapsto a \cdot b$ where the equations $a \cdot x=b, y \cdot a=b$ have unique solutions in $Q$ for all $a, b \in Q$, is called a quasigroup. A loop is a quasigroup with a two-sided identity, $a \cdot e=e \cdot a=a, \forall a \in$ $Q$. A loop $\langle Q, \cdot, e\rangle$ with a smooth functions $\phi(a, b):=a \cdot b$ is called a smooth loop. For $\langle Q, \cdot, e\rangle$ being a local loop with a neutral element $e$ one has the following identity of quasiassociativity

$$
a \cdot(b \cdot c)=(a \cdot b) \cdot l_{(a, b)} c
$$

where $l_{(a, b)}$ is an associator.

Let $\langle\mathrm{QU}(1), *, e\rangle$ be the loop of multiplication by unimodular complex numbers related with the group U(1)as follows: Let $U_{g}=\exp (i g)$, then we define the operation * in $\mathrm{QU}(1)$ by

$$
\begin{aligned}
& U_{g_{1}} * U_{g_{2}}=\mathrm{e}^{i \alpha_{2}\left(g_{1}, g_{2}\right)} U_{g_{12}}, \\
& U_{l\left(g_{1}, g_{2}\right) g_{3}}=\mathrm{e}^{i \Delta\left(g_{1}, g_{2}, g_{3}\right)} U_{g_{3}} \quad U_{g_{1}}, U_{g_{2}} \in \mathrm{QU}(1),
\end{aligned}
$$

where $U_{g_{12}}=U_{g_{1}} U_{g_{2}}$ denotes the conventional multiplication of the elements of the group $\mathrm{U}(1), \alpha_{2}\left(g_{1}, g_{2}\right)$ is a 2-cochain; and $\Delta\left(g_{1}, g_{2}, g_{3}\right)$ will be specified below. Further we assume that the following commutation relations hold:

$$
\begin{aligned}
& \mathrm{e}^{i \alpha_{2}\left(g_{1}, g_{2}\right)} U_{g}=U_{g} \mathrm{e}^{i \alpha_{2}\left(g_{1}, g_{2}\right)}, \\
& \mathrm{e}^{i \Delta\left(g_{1}, g_{2}, g_{3}\right)} U_{g}=U_{g} \mathrm{e}^{i \Delta\left(g_{1}, g_{2}, g_{3}\right)} .
\end{aligned}
$$

For the product of the three elements we find

$$
\begin{aligned}
& U_{g_{1}}\left(U_{g_{2}} U_{g_{3}}\right)=\mathrm{e}^{-i\left(\alpha_{2}\left(g_{1}, g_{23}\right)+\alpha_{2}\left(g_{2}, g_{3}\right)\right)} U_{g_{1}} *\left(U_{g_{2}} * U_{g_{3}}\right), \\
& \left(U_{g_{1}} U_{g_{2}}\right) U_{g_{3}}=\mathrm{e}^{-i\left(\alpha_{2}\left(g_{12}, g_{3}\right)+\alpha_{2}\left(g_{1}, g_{2}\right)\right)}\left(U_{g_{1}} * U_{g_{2}}\right) * U_{g_{3}} .
\end{aligned}
$$

Using the associativity of multiplication in the group $\mathrm{U}(1)$,

$$
U_{g_{1}}\left(U_{g_{2}} U_{g_{3}}\right)=\left(U_{g_{1}} U_{g_{2}}\right) U_{g_{3}},
$$

we obtain

$$
U_{g_{1}} *\left(U_{g_{2}} * U_{g_{3}}\right)=\mathrm{e}^{i \alpha_{3}\left(g_{1}, g_{2}, g_{3}\right)}\left(U_{g_{1}} * U_{g_{2}}\right) * U_{g_{3}},
$$

where the 3 -cocycle $\alpha_{3}\left(g_{1}, g_{2}, g_{3}\right)$ is given by

$$
\alpha_{3}=\alpha_{2}\left(g_{2}, g_{3}\right)-\alpha_{2}\left(g_{12}, g_{3}\right)+\alpha_{2}\left(g_{1}, g_{23}\right)-\alpha_{2}\left(g_{1}, g_{2}\right) \text {. }
$$

From the other hand, the product (23) can be written as

$$
U_{g_{1}} * U_{g_{2}}=U_{g_{1} \cdot g_{2}}
$$

where $g_{1} \cdot g_{2}=g_{1}+g_{2}+\alpha_{2}\left(g_{1}, g_{2}\right)$. Taking into account Eqs. (22), (24) and (26), we find that the triple-product satisfies

$$
U_{g_{1}} *\left(U_{g_{2}} * U_{g_{3}}\right)=\mathrm{e}^{i \Delta\left(g_{1}, g_{2}, g_{3}\right)}\left(U_{g_{1}} * U_{g_{2}}\right) * U_{g_{3}},
$$

Comparing (27) and (29) we conclude that $\Delta\left(g_{1}, g_{2}, g_{3}\right)=\alpha_{3}\left(g_{1}, g_{2}, g_{3}\right)$, and thus, the associator is related to the 3 -cocycle as follows:

$$
l_{\left(g_{1}, g_{2}\right)} g_{3}=g_{3}+\alpha_{3}\left(g_{1}, g_{2}, g_{3}\right) \text {. }
$$

Since the loop QU(1) is isomorphic to the group U(1) if and only if the associator is identity map, one can see that this is equivalent to the vanishing of the 3-cocycle.

Assuming QU(1) to be a local loop, further being called the gauge loop, let us consider the wave function

$$
\Psi(\mathbf{r})=\mathrm{e}^{i g(\mathbf{r})} \psi(\mathbf{r}), \quad \mathrm{e}^{i g} \in \mathrm{QU}(1)
$$

and define the covariant derivative as

$$
D_{\mu} \Psi==\left(\partial_{\mu}-i e A_{\mu}\right) \Psi
$$

where $A_{\mu}$ is a "nonassociative" gauge field. Now applying (23) we find that the wave function $\Psi$ and gauge field $\mathbf{A}$ transform as

$$
\begin{aligned}
& U_{\tilde{g}} * \Psi=\mathrm{e}^{i\left(\tilde{g}+\alpha_{2}(\tilde{g}, g)\right)} \Psi, \\
& \mathbf{A}^{\prime}=\mathbf{A}+\nabla \tilde{g}+\nabla \alpha_{2}(\tilde{g}, g) .
\end{aligned}
$$

It is easy to check that the curvature 2-form, $F=d A$, is invariant under the nonassociative gauge transformations.

Gauge loop in Dirac's monopole problem. - Since the 3 -cocycle $\alpha_{3}\left(\mathbf{r} ; g_{1}, g_{2}, g_{3}\right)$ emerges in the Dirac monopole problem as a general cocycle [17, 18], i.e. it depends on the point $\mathbf{r}$ and the group elements, one has to extend the construction considered above. We generalize it in the following way: Let $\mathcal{Q}$ be the local loop of maps $f_{\gamma}$ : $\mathfrak{M} \mapsto \mathrm{QU}(1), \gamma \in$ String $\mathfrak{M}$. We define the extension String $^{q} \mathfrak{M}$ as the product of String $\mathfrak{M} \otimes \mathcal{Q}$ with

$$
\left(\gamma_{1}, f_{\gamma_{1}}\right)\left(\gamma_{2}, f_{\gamma_{2}}\right)=\left(\gamma_{1} \gamma_{2}, f_{\gamma_{1}} * f_{\gamma_{2}}\right)
$$


where the nonassociative product $f_{\gamma_{1}} * f_{\gamma_{2}}$ is specified as follows: Let $f_{\gamma}$ be the map

$$
f_{\gamma}: \mathbf{r} \mapsto U_{g_{\gamma}}=\exp \left(i \alpha_{1}(\mathbf{r} ; \gamma)\right) \in \mathcal{Q},
$$

then we define

$$
\begin{aligned}
& U_{g_{\gamma_{1}}} * U_{g_{\gamma_{2}}}=U_{g_{\gamma_{1}} \cdot g_{\gamma_{2}}}=\mathrm{e}^{i \alpha_{2}\left(\mathbf{r} ; \gamma_{1}, \gamma_{2}\right)} U_{g_{\gamma_{12}}}, \\
& \left.g_{\gamma_{1}} \cdot g_{\gamma_{2}}=\alpha_{1}\left(\mathbf{r} ; \gamma_{1}\right)+\alpha_{1}\left(\mathbf{r}_{1} ; \gamma_{2}\right)+\sigma(\mathcal{C}, \Sigma)\right),
\end{aligned}
$$

where $\mathbf{r}_{1}=\mathbf{r} \gamma_{1}(1)$, and $\sigma(\mathcal{C}, \Sigma)$ is the contribution of the Dirac string (see Eq. (15)). The 2-cochain $\alpha_{2}$ obyes the following commutation relations:

$$
U_{g_{\gamma}} \mathrm{e}^{i \alpha_{2}\left(\mathbf{r} ; \gamma_{1}, \gamma_{2}\right)}=\mathrm{e}^{i \alpha_{2}\left(\mathbf{r}^{\prime} ; \gamma_{1}, \gamma_{2}\right)} U_{g_{\gamma}}, \quad \mathbf{r}^{\prime}=\mathbf{r} \gamma(1) .
$$

For the triple-product we have the same result as has been obtained above (see Eq. (27)):

$$
U_{g_{\gamma_{1}}} *\left(U_{g_{\gamma_{2}}} * U_{g_{\gamma_{3}}}\right)=\mathrm{e}^{i \alpha_{3}\left(\mathbf{r} ; \gamma_{1}, \gamma_{2}, \gamma_{3}\right)}\left(U_{g_{\gamma_{1}}} * U_{g_{\gamma_{2}}}\right) * U_{g_{\gamma_{3}}}
$$

where $\alpha_{3}=4 \pi \mu$ is the 3 -cocycle.

Dirac's ideas lead naturally to consideration of a quantum mechanics in which the wave function has a nonintegrable (or path-dependent) phase factor. This formalism has been developed by various authors (see, for example 23, 24, 25, 26, 27, 28, 29, 30, 31] and references therein). In general, the path-depndent wave function can be written as

$$
\Psi(\mathbf{r}, \gamma)=\mathrm{e}^{-i e \int_{\gamma} \mathbf{A} \cdot d \mathbf{r}} \psi(\mathbf{r})
$$

where the integration is performed along the path $\gamma$ joining the reference point $\mathbf{r}_{0}$ with a point $\mathbf{r}$, and $\psi(\mathbf{r})$ is a single-valued function.

Here, instead of taking the reference point for the construction of paths, we employ the group String $\mathfrak{M}$ and introduce the path-dependent nonassociative wave function as

$$
\Psi(\mathbf{r}, \gamma)=\mathrm{e}^{i \alpha_{1}(\mathbf{r}, \gamma)} \Psi(\mathbf{r}), \Psi(\mathbf{r}, \gamma) \in \mathcal{Q}, \gamma \in \operatorname{String} \mathfrak{M} .
$$

The realization of the gauge loop $\mathcal{Q}$ in the space of the wave functions $\Psi(\mathbf{r}, \gamma)$ is given by

$$
U_{g_{\gamma^{\prime}}} * \Psi(\mathbf{r} ; \gamma)=\mathrm{e}^{i\left(\alpha_{1}\left(\mathbf{r} ; \gamma^{\prime}\right)+\sigma(\mathcal{C}, \Sigma)\right)} \Psi\left(\mathbf{r} ; \gamma^{\prime} \gamma\right),
$$

where $U_{g_{\gamma^{\prime}}}=\exp \left(i \alpha_{1}\left(\mathbf{r} ; \gamma^{\prime}\right) \in \mathcal{Q}\right.$. Having nonassociative gauge transformations and wave functions, one can define the nonassociative vector potential as above (see Eqs. (31) - (33)).

\section{CONCLUDING REMARKS}

Using of nonassociative structures is unavoidable when the Jacobi identity fails. The emerging difficulties associated with the contradictory nonassociative representations of the gauge group in the presence of the Dirac monopole may be removed by introducing nonassociative gauge transformations, which are related to the theory of quasigroups and loops. In quantum mechanical description of the monopole we deal with nonassociative pathdependent wave function $\Psi(\mathbf{r} ; \gamma)$, where $\gamma \in$ String $\mathfrak{M}$. Returning to the main subject, we see that nonassociative extension of the conventional group $\mathrm{U}(1)$ allows to avoid the Dirac quantization condition and obtain the consistent magnetic monopole theory with an arbitrary magnetic charge.

We close with some remarks about the relevance of this investigation to quantum mechanics and gauge field theory. Since a conventional quantum mechanics requires a linear Hilbert space and associative operators, the Dirac quantization rule is a necessary condition for the consistence of quantum mechanics. Relaxation of this condition implies introducing of a nonassociative algebra of observables, and one must define an equivalent to quantum mechanics without Hilbert space [10, 11, 12, 13, 14]. Non-vanishing 3-cocycles arising in quantum field theory force us to go beyond the standard approach and consider nonassociative generalization of the fibre bundle theory and related nonassociative gauge theories [32, 33]. This work is in progress.
[1] P. A. M. Dirac, Proc. Roy. Soc. Lond. A 133, 60 (1931).

[2] M. Fierz, Helv. Phys. Acta 17, 27 (1944).

[3] A. Peres, Phys. Rev. 167, 1443 (1968).

[4] D. Zwanziger, Phys. Rev. D 3, 880 (1971).

[5] A. Hurst, Ann. Phys. 50, 51 (1968).

[6] H.J. Lipkin, W. I. Weisberg and M. Peskin, Ann. Phys. 53, 203 (1969).

[7] A.I. Nesterov and F. Aceves de la Cruz, Phys. Lett. A 302, 253 (2002).

[8] A.I. Nesterov and F. Aceves de la Cruz, Phys. Lett. A 324, 9 (2004).

[9] A.I. Nesterov and F. Aceves de la Cruz, hep-th/0403146

[10] R. Jackiw, Phys. Rev. Lett. 54, 159 (1985).

[11] B. Grossman, Phys. Lett. B 152, 93 (1985).
[12] Y.-S. Wu and A.Zee, Phys. Lett. B 152, 98 (1985).

[13] D.G. Boulware, S. Deser and B. Zumino, Phys. Lett. B153, 307 (1985).

[14] B. Grossman, Phys. Rev. D 33, 2922 (1986).

[15] A failure of the Jacobi identity also is known in quantum field theory (see ref. [9] and references therein).

[16] J. Wess and B. Bruno, Phys. Lett. B37, 951971.

[17] L.D. Faddeev, Phys. Lett. B 145, 81 (1984).

[18] L.D. Faddeev and A.L. Shatashvili, Teor. Mat. Phys. 60, 206 (1984).

[19] J. Mickelsson, Phys. Rev. Lett. 54, 2379 (1985).

[20] Chein, O., Pflugfelder, H. and Smith, J.D.H. Eds.: Quasigroups and loops: Theory and Applications, (Heldermann Verlag, Berlin, 1990). 
[21] Pflugfelder, H.: Quasigroups and Loops: An Introduction, (Heldermann Verlag, Berlin, 1990).

[22] L.V. Sabinin, Smooth quasigroups and loops, ( Kluwer Academic Publishers, Dordrecht, 1999).

[23] B. S. DeWitt, Phys. Rev. 125, 2189 (1962).

[24] B. J. Belinfante, Phys. Rev. 128, 2832 (1962).

[25] S. Mandelstam, Ann. Phys. 19, 1 (1962).

[26] N. Cabbibo and E. Ferrari, Nuovo Cim. 23, 1147 (1962).

[27] D.K. Ross , Phys. Rev. 181, 2055 (1969).

[28] T. T. Wu and C. N. Yang, Phys. Rev. D 12, 3845 (1975).

[29] T. T. Wu and C. N. Yang, Nucl. Phys. B 107, 365
(1976)

[30] F. Zaccaria, E. C. G. Sudarshan, J. S. Nilsson, N. Mukunda, G. Marmo and A. P. Balachadran, Phys. Rev. D 27, 2327 (1983).

[31] J. R. Ellis, J. Phys. A 23, 65 (1990).

[32] A.I. Nesterov, Principal Q-bundles. In: Non Associative Algebras and Its Applications, ed. R. Costa,H. Cuzzo, Jr. A. Grishkov and L.A. Peresi (Marcel Dekker, New York, 2000).

[33] A.I. Nesterov, Intern. Journ. Theor. Phys. 40, 337 (2001). 